\title{
REFLEXIONES \\ SOBRE COMPETITIVIDAD, EDUCACIÓN Y POBREZA
}

Arturo Maldonado Marmolejo *

\section{RESUMEN}

Hoy en día vivimos un nuevo entorno económico. Las reglas del juego han cambiado. La mundialización implica nuevas prácticas en las empresas, los gobiernos, las instituciones de educación superior, entre otros actores. El mundo global implica más que nada competitividad, es así como se acentúan problemas sistémicos como el desempleo y la pobreza. La competitividad impacta de forma importante al sistema educativo y éste en su conjunto tiene nuevos retos. Redefinir la razón de ser de las universidades es fundamental para abatir el desempleo y la pobreza.

\section{ABSTRACT}

Nowadays we lived new economic surroundings. The rules of the game have changed. The global economic implies new rules and practices in the companies, the governments, the institutions of superior education, among other actors. The new economy implies competitiveness, and both accentuate problematic like unemployment and the poverty. The competitiveness hits of important form to the educative system and this one has new challenges. To redefine the reason of being of the universities is fundamental to lower unemployment and the poverty.

"No hay cambio sin sueño, ni sueño sin esperanza."

\section{Paulo Freire}

Competitividad, desempleo y pobreza son fenómenos de una misma realidad, en un mismo país. México tiene importantes debilidades sistémicas, su historia y sus estadísticas lo confirman. Es "curioso" conocer que nuestra economía es la número diez en términos de su tamaño (producto interno bruto); pero en términos de competitividad, en realidad, ocupamos en el 2005 el lugar número 561 . De igual forma llama la atención la estimación de la Secretaría de Desarrollo Social, quien advierte que 19 millones de mexicanos son pobres urbanos y que el $44 \%$ de la población indígena se encuentra en pobreza extrema.

* Ingeniero Mecánico Electricista de la UNAM, Diplomado en Finanzas y en Formación de Directivos MPYMES por el Tec de Monterrey, Maestría en Administración del ITAM. Experiencia de más de 15 años en la docencia en diversas universidades. Consultor y empresario. Actualmente se desempeña como Profesor de Carrera de Tiempo Completo, Escuela de Ciencias 


\section{Administrativas, Universidad La Salle Pachuca. amaldonado@lasallep.edu.mx}

Con información del Instituto Nacional de Estadística, Geografía e Informática (INEGI), el Instituto Mexicano para la Competitividad, A.C. (IMCO), una firma consultora y experta en análisis económico, nos dicen que si el ingreso en el decil más pobre de la población creciera $3 \%$ anual en términos reales, tardaría 115 años en llegar al nivel de ingresos del decil más rico. A su vez, el Banco Interamericano de Desarrollo (BID) señala que en los países desarrollados, 100\% de los jóvenes de 15 años asisten a la escuela, mientras que en México sólo el $58 \%$ asiste. La inversión en conocimiento en México crece $0.3 \%$ anual, en tanto que en el resto de la OCDE (Organization for Economic Co-operation and Development) crece en promedio $4.8 \%$ anual 2. Finalmente, la Organización Internacional del Trabajo (OIT) señala que como nación, alcanzamos el registro más alto de desempleo juvenil en América Latina: el 9.5\% de los jóvenes de entre 20 y 24 años carece de un empleo formal. El Instituto Mexicano de la Juventud calcula que de los 33.6 millones de jóvenes mexicanos de entre 12 y 29 años de edad, sólo 16 millones tienen empleo formal.

Todas estas cifras y estadísticas invitan a una profunda reflexión. Los discursos, las banderas políticas y la mercadotecnia de los gobiernos no son suficientes para resolver estos problemas históricos. Es evidente que México y los mexicanos hemos perdido competitividad.

Lo anterior no son simplemente números fríos; sino que son cifras conformadas por mexicanos, por mujeres, por ancianos, por jóvenes, por niños. Es por ello que el desarrollo en todos sus aspectos debe de ser una prioridad de cada región, de cada estado y de cada país. La competitividad es la capacidad de las empresas de atraer clientes. Estas son las primeras en competir; pero para ello todos los niveles que las afectan deben de ser competitivos. Los países también deben preocuparse por ser competitivos.

La mundialización obliga a desarrollar localmente a cada región. La competitividad debe ser una ocupación de todos. Nuestro bienestar depende de ella. Una nación competitiva, educa a su niñez y a su juventud; conserva y genera empleo y, en consecuencia, reduce la pobreza. El ámbito local y las vocaciones regionales son fundamentales; pero además se requieren infraestructura adecuada, personal capacitado, proveedores eficientes, escuelas y universidades de calidad, centros de investigación competitivos y gobiernos éticos y con valores que permitan un ambiente adecuado que aseguren la competitividad y la generación de empleo.

La mundialización ha polarizado a México y al mundo. Ha creado y acentuado las diferencias entre el norte y el sur, entre los pobres y los ricos, entre los que acceden a estudios universitarios y los que no tienen ninguna posibilidad de educación, entre los blancos y los otros, entre los hombres y las mujeres. La desigualdad de la distribución de la riqueza, la desigualdad de la educación y el 
conocimiento consolida el poder de las corporaciones. Debe quedarnos claro que la competitividad de las personas, empresas, regiones y países es un asunto de sobrevivencia.

Para avanzar en el tema de la competitividad es necesario observar que el desarrollo se debe dar primero en cada uno de nosotros. Es importante que trabajemos con las personas, en su capacitación, desarrollo y educación. Así la competitividad no será un fin; sino un medio. La competitividad implica el funcionamiento correcto de todos. De todas las partes: la empresa, el personal, los gobiernos, todos debemos funcionar bien. Cada participante debe ser competitivo. En nuestro caso, el factor humano que se conforma de nuestros estudiantes y egresados de las escuelas y universidades también debe aportar su parte. Todos debemos hacer bien el trabajo.

Competitividad es igual a empleo. La competitividad forma un círculo virtuoso. Las empresas competitivas generan ventas, las cuales generan ingresos y empleos bien pagados, que a su vez generan consumo de bienes y servicios y que a su vez generan nuevas empresas e inversiones. Ser competitivo en la producción y en el mercado, genera riqueza y bienestar. Por el contrario, si se interrumpe este círculo, se genera pobreza y marginación, ya que si no somos competitivos simplemente, no vendemos, no invertimos, no generamos empleo.

México es un país de contrastes. Existen diversos tipos de México. De acuerdo con los seminarios de competitividad de la Secretaría del Trabajo y Previsión Social, se destaca que las regiones oeste, noroeste y noreste son las más competitivas de nuestro país, teniendo un nivel comparable en el índice de competitividad a Hong Kong, Israel o Nueva Zelanda. Cabe mencionar que contamos con algunos "conglomerados" exitosos y muy bien definidos como lo son la producción de calzado en León; de lácteos en Torreón; de turismo en Cancún, Los Cabos y Puerto Vallarta; los electrónicos y el software en Guadalajara, Tijuana y Ciudad Juárez; auto-partes en Chihuahua y Saltillo, e incluso biotecnología en Monterrey. En estas regiones se cultiva la educación, el conocimiento y la tecnología.

En contraste las regiones del golfo, centro y sur del país tienen niveles de competitividad equiparables a algunas regiones de Brasil, Perú y Bolivia, respectivamente.

La falta de competitividad, el desempleo y la pobreza son consecuencia natural e inevitable de una nueva economía basada en el conocimiento. Asimismo, tenemos que reconocer que la tecnología y las innovaciones conducen a un proceso de destrucción de prácticas históricamente exitosas. Las jubilaciones, las pensiones, los impuestos, los créditos hipotecarios ya no hacen sentido como antes. Las prácticas nacidas en la revolución industrial no se adaptan al mundo laboral actual por lo que se necesita una nueva forma de entender al mundo. Se necesitan nuevas formas de trabajo y nuevas formas de educar. La educación tradicional, el empleo de por vida y la seguridad económica de las familias son 
cosas del pasado. Debemos aprender a vivir las nuevas reglas del juego de la globalización y la competitividad. A manera de ejemplo, sin la seguridad de una carrera universitaria, una profesión bien remunerada o un empleo en donde jubilarnos, nuestras vidas están en peligro. Hoy nos enfrentamos a la inseguridad en el trabajo y al desempleo, a la competitividad de empresas globales y a la realidad de la pobreza.

Si observamos de manera puntual el tema del desempleo, se identifican diversas causas que explican las nuevas reglas del juego. En principio, internet nos cambió la vida. Con referencias como las de amazon.com en el mundo de las librerías y las casas editoriales o los cambios en las prácticas bancarias (consultas de saldos, pago de servicios, transferencias electrónicas de fondos, entre otros) en todo el mundo, sabremos de los cambios radicales que nos ofrecen las nuevas tecnologías. Internet ha obligado a las empresas a reestructurarse, las reestructuraciones han acabado con escalafones completos de empleados.

Un fenómeno que tiene implicaciones más profundas es la mundialización. El proceso irreversible de mundialización que hoy vivimos ofrece un gran de nuestros estudiantes y egresados de las escuelas y universidades también debe aportar su parte. Todos debemos hacer bien el trabajo.

Competitividad es igual a empleo. La competitividad forma un círculo virtuoso. Las empresas competitivas generan ventas, las cuales generan ingresos y empleos bien pagados, que a su vez generan consumo de bienes y servicios y que a su vez generan nuevas empresas e inversiones. Ser competitivo en la producción y en el mercado, genera riqueza y bienestar. Por el contrario, si se interrumpe este círculo, se genera pobreza y marginación, ya que si no somos competitivos simplemente, no vendemos, no invertimos, no generamos empleo.

México es un país de contrastes. Existen diversos tipos de México. De acuerdo con los seminarios de competitividad de la Secretaría del Trabajo y Previsión Social, se destaca que las regiones oeste, noroeste y noreste son las más competitivas de nuestro país, teniendo un nivel comparable en el índice de competitividad a Hong Kong, Israel o Nueva Zelanda. Cabe mencionar que contamos con algunos "conglomerados" exitosos y muy bien definidos como lo son la producción de calzado en León; de lácteos en Torreón; de turismo en Cancún, Los Cabos y Puerto Vallarta; los electrónicos y el software en Guadalajara, Tijuana y Ciudad Juárez; auto-partes en Chihuahua y Saltillo, e incluso biotecnología en Monterrey. En estas regiones se cultiva la educación, el conocimiento y la tecnología.

En contraste las regiones del golfo, centro y sur del país tienen niveles de competitividad equiparables a algunas regiones de Brasil, Perú y Bolivia, respectivamente.

La falta de competitividad, el desempleo y la pobreza son consecuencia natural e inevitable de una nueva economía basada en el conocimiento. Asimismo, 
tenemos que reconocer que la tecnología y las innovaciones conducen a un proceso de destrucción de prácticas históricamente exitosas. Las jubilaciones, las pensiones, los impuestos, los créditos hipotecarios ya no hacen sentido como antes. Las prácticas nacidas en la revolución industrial no se adaptan al mundo laboral actual por lo que se necesita una nueva forma de entender al mundo. Se necesitan nuevas formas de trabajo y nuevas formas de educar. La educación tradicional, el empleo de por vida y la seguridad económica de las familias son cosas del pasado. Debemos aprender a vivir las nuevas reglas del juego de la globalización y la competitividad. A manera de ejemplo, sin la seguridad de una carrera universitaria, una profesión bien remunerada o un empleo en donde jubilarnos, nuestras vidas están en peligro. Hoy nos enfrentamos a la inseguridad en el trabajo y al desempleo, a la competitividad de empresas globales y a la realidad de la pobreza.

Si observamos de manera puntual el tema del desempleo, se identifican diversas causas que explican las nuevas reglas del juego. En principio, internet nos cambió la vida. Con referencias como las de amazon.com en el mundo de las librerías y las casas editoriales o los cambios en las prácticas bancarias (consultas de saldos, pago de servicios, transferencias electrónicas de fondos, entre otros) en todo el mundo, sabremos de los cambios radicales que nos ofrecen las nuevas tecnologías. Internet ha obligado a las empresas a reestructurarse, las reestructuraciones han acabado con escalafones completos de empleados.

Un fenómeno que tiene implicaciones más profundas es la mundialización. El proceso irreversible de mundialización que hoy vivimos ofrece un grande nuestros estudiantes y egresados de las escuelas y universidades también debe aportar su parte. Todos debemos hacer bien el trabajo.

Competitividad es igual a empleo. La competitividad forma un círculo virtuoso. Las empresas competitivas generan ventas, las cuales generan ingresos y empleos bien pagados, que a su vez generan consumo de bienes y servicios y que a su vez generan nuevas empresas e inversiones. Ser competitivo en la producción y en el mercado, genera riqueza y bienestar. Por el contrario, si se interrumpe este círculo, se genera pobreza y marginación, ya que si no somos competitivos simplemente, no vendemos, no invertimos, no generamos empleo.

México es un país de contrastes. Existen diversos tipos de México. De acuerdo con los seminarios de competitividad de la Secretaría del Trabajo y Previsión Social, se destaca que las regiones oeste, noroeste y noreste son las más competitivas de nuestro país, teniendo un nivel comparable en el índice de competitividad a Hong Kong, Israel o Nueva Zelanda. Cabe mencionar que contamos con algunos "conglomerados" exitosos y muy bien definidos como lo son la producción de calzado en León; de lácteos en Torreón; de turismo en Cancún, Los Cabos y Puerto Vallarta; los electrónicos y el software en Guadalajara, Tijuana y Ciudad Juárez; auto-partes en Chihuahua y Saltillo, e incluso biotecnología en Monterrey. En estas regiones se cultiva la educación, el conocimiento y la tecnología. 
En contraste las regiones del golfo, centro y sur del país tienen niveles de competitividad equiparables a algunas regiones de Brasil, Perú y Bolivia, respectivamente.

La falta de competitividad, el desempleo y la pobreza son consecuencia natural e inevitable de una nueva economía basada en el conocimiento. Asimismo, tenemos que reconocer que la tecnología y las innovaciones conducen a un proceso de destrucción de prácticas históricamente exitosas. Las jubilaciones, las pensiones, los impuestos, los créditos hipotecarios ya no hacen sentido como antes. Las prácticas nacidas en la revolución industrial no se adaptan al mundo laboral actual por lo que se necesita una nueva forma de entender al mundo. Se necesitan nuevas formas de trabajo y nuevas formas de educar. La educación tradicional, el empleo de por vida y la seguridad económica de las familias son cosas del pasado. Debemos aprender a vivir las nuevas reglas del juego de la globalización y la competitividad. A manera de ejemplo, sin la seguridad de una carrera universitaria, una profesión bien remunerada o un empleo en donde jubilarnos, nuestras vidas están en peligro. Hoy nos enfrentamos a la inseguridad en el trabajo y al desempleo, a la competitividad de empresas globales y a la realidad de la pobreza.

Si observamos de manera puntual el tema del desempleo, se identifican diversas causas que explican las nuevas reglas del juego. En principio, internet nos cambió la vida. Con referencias como las de amazon.com en el mundo de las librerías y las casas editoriales o los cambios en las prácticas bancarias (consultas de saldos, pago de servicios, transferencias electrónicas de fondos, entre otros) en todo el mundo, sabremos de los cambios radicales que nos ofrecen las nuevas tecnologías. Internet ha obligado a las empresas a reestructurarse, las reestructuraciones han acabado con escalafones completos de empleados.

Un fenómeno que tiene implicaciones más profundas es la mundialización. El proceso irreversible de mundialización que hoy vivimos ofrece un gran potencial de crecimiento para los que tienen capacidad competitiva; pero a la vez excluye a todos los demás.

Con lo anterior, el trabajo tiene un nuevo significado para todos. Para los estudiantes, para los profesores, para las universidades, para las empresas, para los sindicatos, para el gobierno. Para todos. El empleo debe ser sustituido por modelos definidos por una nueva sensibilidad hacia el cliente, las innovaciones tecnológicas y las culturas globalizadas. En adición, los expertos en mercadotecnia aseguran que la competencia entre las empresas es cada día más agresiva y hay mayor necesidad de ser competitivos como personas, empresas y regiones. Hoy competimos no sólo con los de enfrente o los de al lado, competimos con todo el mundo.

Con base en el estudio realizado por Fernando Flores (2002), sobre la obsolescencia de las carreras como forma de desarrollo profesional, se señala que "con el deceso de las profesiones, dos formas de vida laboral significativamente 
diferentes están surgiendo en las sociedades occidentales. Por un lado la forma 'conectada' rápida, con redes globales, centrada en proyectos, está surgiendo en los llamados conglomerados. Por otro lado está surgiendo otra clase de vida laboral en formas novedosas a los bienes sociales tradicionales de una profesión: el espíritu emprendedor"3. Esta propuesta genera una seria reflexión sobre el papel de la educación tradicional de escuelas y universidades.

A medida que va desapareciendo el empleo tradicional, están surgiendo nuevas formas de trabajo que desplazan la tradicional figura del empleo.

Por ejemplo, para un emprendedor, la vida tiene sentido cuando va más allá de la expresión de sí mismo, ve más allá de su persona y trata de renovar la vida de la colectividad mediante la creación de un nuevo producto o servicio, un nuevo logro económico o político, un acontecimiento cultural o deportivo y un nuevo servicio social. Observemos que se puede ser emprendedor en muchos aspectos de la vida, además de aquellos que crean una nueva empresa.

México es un país de jóvenes. El reto de la competitividad es un desafío para los jóvenes de hoy, en términos de generar los empleos requeridos; si queremos que se inserten a actividades productivas de manera formal. México se enfrenta al reto de la competitividad, la mundialización y la apertura comercial.

El capital humano es fundamental en las empresas, por ello deben fortalecer la capacitación, la vinculación de las universidades con las empresas y los sectores productivos. Los jóvenes son parte central de la productividad en especial, porque en algunos años nos acercaremos a los máximos históricos en cuanto a población, población económicamente activa y población joven.

Nuestros estudiantes no requieren que les demos una formación siguiendo las disciplinas y los métodos tradicionales. Necesitan que se les enseñe la capacidad para aprender, emprender, colaborar y transformar por sí mismos. Es decir, que se les forme para que sepan cómo encontrar oportunidades de crear algún tipo de valor para ellos y para la comunidad. Con esta reorientación lo que se pretende es educarlos como a personas que hacen ofertas y no como pasivos "tomadores de pedidos", totalmente reactivos, esperando a que "alguien" les diga qué hacer.

Una persona es emprendedora si observa con curiosidad y busca una mejor realidad. Saiolan 4, el centro de incubación de empresas del corporativo Mondragón, al norte de España, propone que "emprender es empezar una cosa que implica trabajo o presenta dificultades y equivale a afrontar, abrir camino, actuar, ejecutar, construir, hacer, acertar y errar. Es decir avanzar en la vida".

En consecuencia, nuestros jóvenes, tanto de escuelas públicas como privadas, deben aprender a hacer propuestas, a escuchar de otro modo, a relacionarse, a coordinar compromisos, a crear confianza y mantenerla. Tendrán que aprender algunas de las competencias que hasta ahora sólo se atribuían a los líderes. 
Por ello es necesario consolidar nuevas prácticas y hábitos de trabajo y de estudio que nos permitan avanzar en la transformación social y económica que queremos. Para transformar debemos empezar por transformarnos a nosotros. Debemos transformar nuestras prácticas, costumbres y hábitos en los diversos roles que coexisten sobre todo en nuestras escuelas y universidades: alumnos y profesores. Para el mundo de hoy, debemos pensar en nuevas escuelas, nuevas universidades, nuevos profesores y nuevos alumnos. Tenemos que desarrollar y reorientar nuestros aprendizajes.

Y por el bien de todos, primero los pobres. Casi la mitad de la población en México vive en la pobreza y de ésta, casi la mitad, sobrevive a la pobreza extrema. En el caso del estado de Hidalgo, este gran estado, rico en historia y tradición, se conoce por la autoridad en la materia que este estado es uno de los nueve con mayor nivel de pobreza y marginación, según Cabildo (2005) 5. Por ello sería importante pensar y actuar en las "comunidades que menos tienen". El no ser competitivos nos afecta. Nuestra pobreza es el costo de dicha falta de competitividad.

Para familiarizarnos en el tema de pobreza, conozcamos algunas cifras que nos dan un bosquejo de la misma. Se tiene que hay en el mundo 1,200 millones de personas que sobreviven con menos de un dólar diario. En América latina existen cerca de 250 millones de pobres, bajo esta referencia. El Banco Mundial define la pobreza a un "dólar por día", es decir, se establece un "umbral de la pobreza" a un dólar por día por persona. El "índice de pobreza humana" (IPH) se basa en "las dimensiones más básicas de privación: un lapso de vida breve, falta de educación básica y falta de acceso a recursos públicos y privados." 6

La pobreza en México se acentúa desde los años 80, el empobrecimiento de los pequeños campesinos y la reducción del poder adquisitivo. La mundialización agudiza la ola creciente de la pobreza en México desde la firma del Tratado de Libre Comercio de América del Norte. Con base en el Informe de Desarrollo Humano, del Programa de Desarrollo de las Naciones Unidas (PNUD) en 1997, según Chossudovsky (2005) el índice de la pobreza, sitúa a nuestro país con un nivel de pobreza (porcentaje de la población bajo el nivel de pobreza) en el orden del 10.9, con niveles similares a Tailandia, 11.7; Colombia, 10.7; Jordania, 10.9 y Jamaica, 12.1; y afortunadamente por debajo de otros países como Nicaragua, 27.2; Irak, 30.7; Ruanda, 37.9 y Nigeria, 41.6 . 7

De manera paralela y con la finalidad de plantear alternativas de solución a las problemáticas anteriores, conviene recordar la razón de ser de las universidades. Es decir pocas instituciones u organismos tienen ese grado de conciencia crítica y la capacidad de pensar, razonar y reflexionar como las propias universidades, tanto públicas como privadas. Es justo mencionar también la necesidad de intentar aprender un poco de esa visión del señor De La Salle, quien sin duda fue, es y será un ejemplo a seguir en el tema "asociados en el servicio educativo de los pobres". Esta vocación por los pobres se remonta a 1694, cuando Juan Bautista De la Salle y doce de sus compañeros se asociaron para dar una educación 
cristiana a los pobres. Por más de 300 años "indivisa manent" ha pasado la prueba del tiempo.

En las universidades debemos entender la competitividad, el desempleo y la pobreza de forma diferente. Las escuelas y universidades, públicas y privadas, tenemos un compromiso fundamental. Pocas instituciones pueden asumir estos retos. Es por ello que debemos hacer bien nuestro trabajo, es decir, debemos hacer docencia de calidad, en su caso, investigación de calidad y evidentemente difusión de calidad. Por la competitividad de nuestras regiones, la conservación y generación de más y mejores empleos y la reducción de los niveles indignos de pobreza, las escuelas y las universidades debemos hacer trabajo de calidad y no cantidad. La calidad no podemos abandonarla, no podemos formar futuros desempleados.

La educación es la base de la competitividad, del empleo y del bienestar. Como lo señala el Dr. Alberto Equihua Zamora (2005), Director de Análisis y Estrategia de la Confederación Patronal de la República Mexicana (COPARMEX), el $10 \%$ de la población más pobre en México tiene apenas una escolaridad de 3 años, en tanto el $10 \%$ de la población más rica tiene una escolaridad promedio de 12 años 8 . Esto último es un claro ejemplo de formar un círculo virtuoso; en contraste, la población más pobre probablemente forme un círculo vicioso difícil de romper. La relación entre competitividad y educación es clara y preocupante.

Como lo señala Castro Silva (2002)9, en su conferencia Sentido y Misión de la Universidad Latinoamericana, "La universidad debe convertirse en un elemento de apoyo al desarrollo de un sistema educativo que permite al individuo educarse toda la vida, procurando igualdad de oportunidades y de resultados de calidad equivalente. Para este efecto, deberá observar un rol más protagónico y activo en la retroalimentación, la transformación y orientación del conjunto del sistema para responder a los nuevos requerimientos de calidad y equidad".

Necesitamos ponernos de acuerdo, definir qué queremos hacer. Las universidades necesitan preparar a los jóvenes para que alcancen empleo y se integren a las empresas y sus necesidades. Necesitamos ponernos de acuerdo en el perfil de lo que requieren las empresas, qué carreras se necesitan, el perfil del egresado, quiénes tienen empleo, qué licenciaturas necesitan las regiones, qué tipo de habilidades y conocimientos necesitan los negocios, cuáles pueden tener un empleo, qué nivel de ingresos tendrán, qué nivel de competencia se requiere. Por ello reconocemos que las universidades se deben vincular con las realidades de cada región.

Si la pobreza se ha agudizado; entonces, el reto para todas las universidades mexicanas, si éstas están concientes de la realidad nacional, es convertirse en un instrumento para erradicar la desigualdad social y la pobreza, entre otras problemáticas sistémicas. 
En este orden de ideas se identifican las siguientes líneas de acción a seguir por las instituciones de educación superior: buscar ser accesibles al mayor número de personas posibles; formar a nuestros alumnos en la justicia y en la solidaridad; contribuir a la transformación del contexto social. De forma puntual las universidades de inspiración cristianas plantean, según Govela (2002)10 , que "no se puede pretender por sí solo conseguir un acercamiento educativo y una acción eficaz ante todas las formas actuales de pobreza. Es importante que establezcan relaciones y colaboren con otras organizaciones y otras instancias (políticas, sociales, religiosas, entre otras) que trabajen en resolver problemas de los pobres en los niveles local, regional o internacional".

Según Gorostiaga (2002)11, en relación a las universidades destaca que:

1) La universidad tiene una gran oportunidad para convertirse en un instrumento eficaz y poderoso del servicio educativo a los pobres, a pesar de que aparentemente no tiene una vocación natural hacia ello.

2) Para realizar esta conversión es necesario resolver algunas tensiones entre el servicio y su accesibilidad, de forma que la primera manera de servir efectivamente a la educación, sea aceptándolos en sus aulas.

3) La universidad no puede ser un instrumento aislado para el servicio educativo de los po- bres, se requiere un sistema de instituciones, donde se combinen en proporción adecuada las de educación superior, las de educación básica y media, y las de servicio directo a los más pobres.

El reto de ser competitivos, generar empleo y abatir la pobreza está ahí. Las escuelas en sus diversos niveles y las instituciones de educación superior deben de reflexionar profundamente en las diversas formas que tienen para erradicar la pobreza y la desigualdad educativa.

No es suficiente tener más alumnos y más egresados, México ha aumentado sus niveles de desigualdad y de pobreza. El reto de las universidades es usar el conocimiento como un elemento democratizador formando al recurso humano para el desarrollo sostenible. Debemos cambiar los paradigmas de las universidades y de la educación al dejar de ser una herramienta de movilización social para acceder a riquezas y poder. Las universidades deben vincularse para crear una nueva riqueza en los egresados de forma sostenible, equitativa y democrática, como lo propone Xavier Gorostiaga (2002) en el documento "En busca del eslabón perdido entre educación y desarrollo".

Con la finalidad de aprovechar los esfuerzos que ya se han realizado y centrados en el tema de competitividad y pobreza, quiero señalar las líneas centrales propuestas por el Proyecto Educativo Regional Lasallista (PERLA) en lo concerniente a la democratización del conocimiento: 1) comprensión de la realidad; 2) una universidad que escucha; 3) universidad y sociedad del 
conocimiento; 4) el desarrollo del talento humano y 5) formación de líderes y estructura para democratizar el conocimiento.

Como lo propone Oscar Azmitia (2002)12 la opción por los más empobrecidos es la democratización del conocimiento. Él afirma que la educación universitaria tiene dimensiones de un proyecto social pues debe revertir la injusticia social, dando oportunidades de superación a aquellos que no pueden asistir a la universidad y a quienes trabajan por asistir a ella. Por ellos los centros de estudios superiores están llamados a atender a la población sin acceso de espacios y programas educativos formales y no formales para que las poblaciones excluidas mejoren sus condiciones de vida y sean sujetos de su propio destino; difundir el conocimiento sobre el marco teórico que implica la democratización del conocimiento y sistematizar experiencias sobre el acceso a los más empobrecidos. Asimismo, las actitudes para la transformación, para la democracia, no se adquieren, ni se otorgan; se forjan. Se forjan a través de la educación. El papel de la universidad es de vital importancia en esa transformación, impulsora del cambio social, la transformación de la persona integral conectada con su realidad.

Como podemos ver la transformación de la región no es un tema menor, no es tampoco una teoría que "suena bien", no es un concepto "de moda". Sin embargo, estudiar la competitividad, el desempleo o la pobreza no es suficiente.

La transformación de las regiones se dará en función de nuestros propios cambios. Cómo es posible esperar algo diferente si siempre hacemos lo mismo. La transformación personal nos llevará a la transformación de la región, en donde toda la comunidad esté, en realidad, trabajando en favor de los menos favorecidos. Entonces sí, por el bien de todos, primero los pobres. Los mexicanos estamos obligados a elevar nuestra competitividad, abatir el desempleo y la pobreza. El tiempo ha pasado. México está a menos de medio siglo de llegar a sus máximos de población, total y económicamente activa. En diez años estaremos preparando en las universidades a nuestra mayor población económicamente activa. Si no resolvemos los retos de la competitividad, el desempleo y la pobreza estaremos condenados a vivir en una economía caracterizada no sólo por la falta de competitividad, eso qué importa, estaremos asegurándoles a nuestros jóvenes y niños, opciones de desempleo y de pobreza. Así ellos, el futuro de México, será una población adulta con serios problemas económicos. Las estadísticas son afirmaciones que no dejan espacio a interpretaciones.

En los 70 decíamos: ¡México, pobre!

En los 80: ¡México, pobre!

En los 90: ¡México, pobre!

Pero para los siguientes años diremos: ¡Pobre México! 


\section{NOTAS}

1 Organization for Economic Co-operation and Development (OCDE) (2005). World Competitiveness Yearbook 2005, OCDE. Recuperado 19, octubre, 2005.

2 México Competitivo (2005). La situación de la competitividad en México, Secretaría del Trabajo y Previsión Social.

3 Flores, Fernando (2002) "El final de las profesiones, nuevas formas de trabajo".

4 SAIOLAN (2003) Competencia Iniciativa Emprendedora. Centro de Empresas e Innovación de Mondragón, S. A.

5 Cabildo, Miguel. (2005). Registra Sedesol "pobreza alta" en 9 estados. "Revista Proceso".

6 Chossudovsky, Michel. (2002). Falsedades globales. Cómo el Banco Mundial y el PNUD (Programa de Desarrollo de las Naciones Unidas) deforman las cifras sobre la pobreza global.

7 Chossudovsky, Michel (2005). "Falsedades globales Cómo el Banco Mundial y el PNUD deforman las cifras sobre la pobreza global".

8 México Competitivo (2005). La situación de la competitividad en México, Secretaría del Trabajo y Previsión Social.

9 Castro Silva, Eduardo. (2002). Sentido y misión de la universidad latinoamericana. Reflexiones sobre educación superior.

10 Govela Gutiérrez, Andrés. (2002) Circular 447, sugerencia del capítulo 43 general. La educación superior lasallista y la renovada opción por los pobres en América Latina y el Caribe. Reflexiones sobre educación superior.

11 Govela Gutiérrez, Andrés. (2002). La educación superior lasallista y la renovada opción por los pobres en América Latina y el Caribe. Reflexiones sobre educación superior.

12 Azmitia, Oscar. (2002). La contribución de la educación superior lasallista al proyecto educativo regional latinoameri- cano. Reflexiones sobre educación superior

\section{REFERENCIAS}


Organization for Economic Co-operation and Development (OCDE) (2005). World Competitiveness Yearbook 2005, OCDE. Recuperado 19, octubre, 2005. Bogota, Colombia: Información enviada por correo electrónico.

FLORES, Fernando, 2002 El final de las profesiones, nuevas formas de trabajo. México, Distrito Federal: Información enviada por correo electrónico.

SAIOLAN, 2003, Competencia Iniciativa Emprendedora. Centro de Empresas e Innovación de Mondragón, S. A. Mondragón, España: Información enviada por correo electrónico.

CABILDO, Miguel, 2005, Revista Proceso. México, Distrito Federal: Proceso.

CHOSSUDOVSKY, Michel, 2002, Falsedades globales. Cómo el Banco Mundial y el PNUD (Programa de Desarrollo de las Naciones Unidas) deforman las cifras sobre la pobreza global. Otawa, Canada: Información enviada por correo electrónico.

CASTRO, Eduardo, 2002, Reflexiones sobre educación superior. Santiago, Chile: Región Latinoamericana Lasallista.

GOVELA, Andrés, 2002, Reflexiones sobre educación superior. Santiago, Chile: Región Latinoamericana Lasallista.

AZMITIA, Oscar, 2002, Reflexiones sobre educación superior. Santiago, Chile: Región Latinoamericana Lasallista. 